\title{
The State of OA: A large-scale analysis of the prevalence and impact of Open Access articles
}

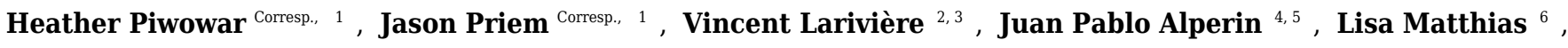 \\ Bree Norlander ${ }^{7,8}$, Ashley Farley ${ }^{7,8}$, Jevin West ${ }^{7}$, Stefanie Haustein ${ }^{3,9}$ \\ 1 Impactstory, Sanford, USA \\ 2 École de bibliothéconomie et des sciences de l'information, Université de Montréal, Montréal, QC, Canada \\ 3 Observatoire des Sciences et des Technologies (OST), Centre Interuniversitaire de Recherche sur la Science et la Technologie (CIRST),, Université du \\ Québec à Montréal, Montréal, QC, Canada \\ 4 Canadian Institute for Studies in Publishing, Simon Fraser University, Vancouver, BC, Canada \\ 5 Public Knowledge Project \\ 6 Scholarly Communications Lab, Simon Fraser University, Vancouver, Canada \\ 7 Information School, University of Washington, Seattle, USA \\ 8 FlourishOA \\ 9 School of Information Studies, University of Ottawa, Ottawa, ON, Canada \\ Corresponding Authors: Heather Piwowar, Jason Priem \\ Email address: heather@impactstory.org, jason@impactstory.org
}

Despite growing interest in Open Access (OA) to scholarly literature, there is an unmet need for largescale, up-to-date, and reproducible studies assessing the prevalence and characteristics of OA. We address this need using oaDOI, an open online service that determines OA status for 67 million articles.

We use three samples, each of 100,000 articles, to investigate OA in three populations: 1) all journal articles assigned a Crossref DOI, 2) recent journal articles indexed in Web of Science, and 3) articles viewed by users of Unpaywall, an open-source browser extension that lets users find OA articles using oaDOI.

We estimate that at least $28 \%$ of the scholarly literature is OA (19M in total) and that this proportion is growing, driven particularly by growth in Gold and Hybrid. The most recent year analyzed (2015) also has the highest percentage of OA (45\%). Because of this growth, and the fact that readers disproportionately access newer articles, we find that Unpaywall users encounter OA quite frequently: $47 \%$ of articles they view are OA. Notably, the most common mechanism for OA is not Gold, Green, or Hybrid OA, but rather an under-discussed category we dub Bronze: articles made free-to-read on the publisher website, without an explicit Open license.

We also examine the citation impact of OA articles, corroborating the so-called open-access citation advantage: accounting for age and discipline, OA articles receive $18 \%$ more citations than average, an effect driven primarily by Green and Hybrid OA. We encourage further research using the free oaDOI service, as a way to inform OA policy and practice. 


\section{The State of OA: A large-scale analysis of the 2 prevalence and impact of Open Access articles}

3 Piwowar, Priem, Larivière, Alperin, Matthias, Norlander, Farley, West, Haustein

- Heather Piwowar. 0000-0003-1613-5981

- Impactstory, Sanford USA

- Jason Priem. 0000-0001-6187-6610

- Impactstory, Sanford USA

- Vincent Larivière. 0000-0002-2733-0689

- École de bibliothéconomie et des sciences de l'information, Université de Montréal, Montréal, QC, Canada.

- Observatoire des Sciences et des Technologies (OST), Centre Interuniversitaire de Recherche sur la Science et la Technologie (CIRST), Université du Québec à Montréal, Montréal, QC. Canada

- Juan Pablo Alperin 0000-0002-9344-7439

- Canadian Institute for Studies in Publishing, Simon Fraser University, Vancouver, BC, Canada

- Public Knowledge Project

- Lisa Matthias. 0000-0002-2612-2132

- Scholarly Communications Lab, Simon Fraser University, Vancouver, BC, Canada

- Bree Norlander. 0000-0002-0431-4221

- University of Washington, Information School, Seattle, USA

- FlourishOA

- Ashley Farley. 0000-0001-9310-6944

- University of Washington, Information School, Seattle, USA

- FlourishOA

- Jevin West. 0000-0002-4118-0322

- University of Washington, Information School, Seattle, USA

- Stefanie Haustein. 0000-0003-0157-1430

- School of Information Studies, University of Ottawa, Ottawa, ON Canada

- Observatoire des Sciences et des Technologies (OST), Centre Interuniversitaire de Recherche sur la Science et la Technologie (CIRST), Université du Québec à Montréal, Montreal, QC Canada

\section{Abstract}

Despite growing interest in Open Access (OA) to scholarly literature, there is an unmet need for large-scale, up-to-date, and reproducible studies assessing the prevalence and characteristics of OA. We address this need using oaDOI, an open online service that determines OA status for 67 million articles. We use three samples, each of 100,000 articles, to investigate OA in three populations: 1) all journal articles assigned a Crossref DOI, 2) recent journal articles indexed in Web of Science, and 3) articles viewed by users of Unpaywall, an open-source browser extension that lets users find OA articles using oaDOI. We estimate that at least $28 \%$ of the scholarly literature is OA (19M in total) and that this proportion is growing, driven particularly by growth in Gold and Hybrid. The most recent year analyzed (2015) also has the highest percentage of OA $(45 \%)$. Because of this growth, and the fact that readers disproportionately access newer articles, we find that Unpaywall users encounter OA quite frequently: $47 \%$ of articles they view are OA. Notably, the most common mechanism for OA is not Gold, Green, or 
46 on the publisher website, without an explicit Open license. We also examine the citation impact

47 of OA articles, corroborating the so-called open-access citation advantage: accounting for age

48 and discipline, OA articles receive 18\% more citations than average, an effect driven primarily by

49 Green and Hybrid OA. We encourage further research using the free oaDOI service, as a way to

50 inform OA policy and practice.

\section{Introduction}

52 The movement to provide open access (OA) to all research literature is now over fifteen years

53 old. In the last few years, several developments suggest that after years of work, a sea change

54 is imminent in OA. First, funding institutions are increasingly mandating OA publishing for

55 grantees. In addition to the US National Institutes of Health, which mandated OA in $2008^{1}$, the

56 Bill and Melinda Gates Foundation ${ }^{2}$, the European Commission ${ }^{3}$, the US National Science

57 Foundation $^{4}$, and the Wellcome Trust ${ }^{5}$, among others, have made OA diffusion mandatory for

58 grantees. Second, several tools have sprung up to build value atop the growing OA corpus.

59 These include discovery platforms like ScienceOpen and 1Science, and browser-based

60 extensions like the Open Access Button, Canary Haz, and Unpaywall. Third, Sci-Hub (a website

61 offering pirate access to full text articles) has built an enormous user base, provoking newly

62 intense conversation around the ethics and efficiency of paywall publishing (Bohannon, 2016;

63 Greshake, 2017). Academic social networks like ResearchGate and Academia.edu now offer

64 authors an increasingly popular but controversial solution to author self-archiving (Björk, 2016).

65 Finally, the increasing growth in the cost of toll-access subscriptions, particularly via so-called

66 "Big Deals" from publishers, has begun to force libraries and other institutions to initiate large-

67 scale subscription cancellations; recent examples include Caltech, the University of Maryland,

68 University of Konstanz, Université de Montréal, and the national system of Peru (Université de

69 Montréal, 2017; Schiermeier \& Mega, 2017; Anderson, 2017a; Universitat Konstanz, 2014). As

70 the toll-access status quo becomes increasingly unaffordable, institutions are looking to OA as

71 part of their "Plan B" to maintain access to essential literature (Antelman, 2017).

72 Open access is thus provoking a new surge of investment, controversy, and relevance across a

73 wide group of stakeholders. We may be approaching a moment of great importance in the

74 development of $\mathrm{OA}$, and indeed of the scholarly communication system. However, despite the

75 recent flurry of development and conversation around OA, there is a need for large-scale, high-

76 quality data on the growth and composition of the OA literature itself. In particular, there is a

77 need for a data-driven "state of OA" overview that is a) large-scale, b) up-to-date, and c)

78 reproducible. This paper attempts to provide such an overview, using a new open web service

79 called oaDOI that finds links to legally-available OA scholarly articles. ${ }^{6}$ Building on data provided

80 by the oaDOI service, we answer the following questions:

11 https://publicaccess.nih.gov/index.htm

22 http://www.gatesfoundation.org/How-We-Work/General-Information/Open-Access-Policy

33 http://ec.europa.eu/research/participants/data/ref/h2020/grants_manual/hi/oa_pilot/h2020-hi-

4 oa-pilot-guide_en.pdf

54 https://www.nsf.gov/pubs/2015/nsf15052/nsf15052.pdf

65 https://wellcome.ac.uk/press-release/wellcome-trust-strengthens-its-open-access-policy

76 In the interest of full disclosure, it should be noted that two of the authors of the paper are the co-

8 founders of ImpactStory, the non-profit organization that developed oaDOI. 
81

82

83

84

85

86

87

1. What percentage of the scholarly literature is $O A$, and how does this percentage vary according to publisher, discipline, and publication year?

2. Are OA papers more highly-cited than their toll-access counterparts?

The next section provides a brief review of the background literature for this paper, followed by a description of the datasets and methods used, as well as details on the definition and accuracy of the oaDOI categorization. Results are then presented, in turn, for each research question, and are followed by a general discussion and conclusions.

\section{Literature review}

Fifteen years of OA research have produced a significant body of literature, a complete review of which falls outside the scope of this paper (for recent, in-depth reviews, see Tennant et al. [2016] and Mckiernan et al. [2016]). Here we instead briefly review three major topics from the OA literature: defining $O A$ and its subtypes, assessing the prevalence of $O A$, and examining the relative citation impact of $O A$.

Despite the large literature on OA, the term itself remains "somewhat fluid" (Antelman, 2004), making an authoritative definition challenging. The most influential definition of OA comes from the 2002 Budapest Open Access Initiative (BOAI), and defines OA as making content both free to read and free to reuse, requiring the opportunity of OA users to "crawl [articles] for indexing, pass them as data to software, or use them for any other lawful purpose." In practice, the BOAI definition is roughly equivalent to the popular "CC-BY" Creative Commons license (Creative Commons, n.d.). However, a number of other sources prefer a less strict definition, requiring only that OA "makes the research literature free to read online" (Willinsky, 2003), or that it is "digital, online, [and] free of charge." (Matsubayashi, 2009). Others have suggested it is more valuable to think of OA as a spectrum (Chen and Olijhoek, 2016).

Researchers have identified a number of subtypes of OA; some of these have near-universal support, while others remain quite controversial. We will not attempt a comprehensive list of these, but instead note several that have particular relevance for the current study.

- Libre OA (Suber, 2008): extends user's rights to read and also to reuse literature for purposes like automated crawling, archiving, or other purposes. The Libre OA definition is quite similar to the BOAI definition of OA.

- Gratis OA (Suber, 2008): in contrast to Libre, Gratis extends only rights to read articles.

- Gold OA: articles are published in an "OA journal," a journal in which all articles are open directly on the journal website. In practice, OA journals are most often defined by their inclusion in the Directory of Open Access Journals (DOAJ) (Archambault et al., 2014; Gargouri et al., 2012).

- Green OA: Green articles are published in a toll-access journal, but self-archived in an OA archive. These "OA archives" are either disciplinary repositories like ArXiv, or "institutional repositories (IRs) operated by universities, and the archived articles may be either the published versions, or electronic preprints (Harnad et al. 2008). Most Green OA articles do not meet the BOAI definition of OA since they do not extend reuse rights (making them Gratis OA). 
- Hybrid OA: articles are published in a subscription journal but are immediately free to read under an open license, in exchange for an an article processing charge (APC) paid by authors (Walker, 1998; Laakso and Björk, 2013)

- Delayed OA: articles are published in a subscription journal, but are made free to read after an embargo period (Willinsky, 2009; Laakso and Björk, 2013).

- Academic Social Networks (ASN): Articles are shared by authors using commercial online social networks like ResearchGate and Academia.edu. While some include these in definitions of OA (Archambault et al. 2013; Björk 2016b), others argue that content shared on ASNs is not OA at all. Unlike Green OA repositories, ASNs do not check for copyright compliance, and therefore as much as half their content is illegally posted and hosted (Jamali, 2017). This raises concerns over the persistence of content, since, as was the case in October 2017, publishers can and do issue large-scale takedown notices to ASN ordering the removal of infringing content (Chawla, 2017). Others have raised questions about the sustainability and ethics of ASN services themselves (Fortney and Gonder, 2015). Due to these concerns, and inconsistent support from the literature, we exclude ASN-hosted content from our definition of $\mathrm{OA}^{7}$

- "Black OA": Articles shared on illegal pirate sites, primarily Sci-Hub and LibGen. Although (Björk 2017) labels these articles as a subtype of OA, the literature has nearly no support for including Sci-Hub articles in definitions of OA. Given this, we exclude Sci-Hub and LibGen content from our definition of OA.

Based on the consensus (and in some cases, lack of consensus) around these definitions and subtypes, we will use the following definition of $O A$ in the remainder of this paper: OA articles are free to read online, either on the publisher website or in an OA repository.

\section{Prevalence of OA}

Many studies have estimated what proportion of the literature is available OA, including Björk et al. (2010), Laakso et al. (2011), Laakso and Björk (2012), Gargouri, Lariviere, Gingras, Carr, and Harnad (2012), Archambault (2013), Archambault et al. (2014), and Chen (2014). We are not aware of any studies since 2014 . The most recent two analyses estimate that more than $50 \%$ of papers are now freely available online, when one includes both OA and ASNs. Archambault et al. (2014), the most comprehensive study to date, estimates that of papers published between 2011 and $2013,12 \%$ of articles could be retrieved from the journal website, $6 \%$ from repositories, and $31 \%$ by other mechanisms (including ASNs). Archambault et al. (2014) also found that the availability of papers published between 1996 and 2011 increased by $4 \%$ between April 2013 and April 2014, noting that "backfilling" is a significant contributor to green OA. Their discipline-level analysis confirmed the findings of other studies, that the proportion of OA is relatively high in biomedical research and math, while notably low in engineering, chemistry, and the humanities.

This Archambault et al. (2014) study is of particular interest because it used automated web scraping to find and identify OA content; most earlier efforts have relied on laborious manual

\footnotetext{
7 Repositories that were included are those covered by the Bielefeld Academic Search Engine (BASE) in May 2017. A full listing of repositories can be found on their website at: https://www.basesearch.net/about/en/about_sources_date.php?menu=2\&submenu=1
} 
160

161

162

163

164

165

166

167

168

169

170

171

checking of the DOAJ, publisher webpages, Google, and/or Google Scholar (though see Hajjem, Harnad, and Gingras [2006] for a notable early exception). By using automated methods, Archambault et al. were able to sample hundreds of thousands of articles, greatly improving statistical power and supporting more nuanced inferences. Moreover, by creating a system that indexes OA content, they address a major concern in the world of OA research; as Laakso (2011) observes: "A major challenge for research...has been the lack of comprehensive indexing for both OA journals and their articles." The automated system of Archambault et al. (2014) is very accurate -- it only misclassifies a paper as OA $1 \%$ of the time, and finds about $75 \%$ of all OA papers that exist online, as per Archambault, Côté, Struck, \& Voorons (2016). However, the algorithm is not able to distinguish Gold from Hybrid OA. More problematically for researchers, the database used in the study is not open online for use in follow-up research. Instead, the data has since been used to build the commercial subscription-access database 1 science. ${ }^{8}$

\section{The Open Access Citation Advantage}

173 Several dozen studies have compared the citation counts of OA articles and toll-access articles. Most of these have reported higher citation counts for OA, suggesting a so-called "open access citation advantage" (OACA); several annotated bibliographies have been created to track this literature (SPARC Europe, 2015; Wagner, 2010; Tennant, n.d.). The OACA is not universally supported. Many studies supporting the OACA have been criticised on methodological grounds (Davis \& Walters, 2011), and an investigation using the randomized-control trial method failed to find evidence of an OACA (Davis, 2011). However, recent investigations using robust methods have continued to observe an OACA. For instance, McCabe and Snyder (2014) used a complex statistical model to remove confounding effects of author selection (authors may selectively publish their higher-impact work as OA), reporting a small but meaningful $8 \%$ OACA. Archambault et al. (2014) describe a 40\% OACA in a massive sample of over one million articles using field-normalized citation rates. Ottaviani (2016) used a natural experiment as articles (not selected by authors) emerged from embargoes to become OA, and reports a 19\% OACA excluding the author self-selection bias for older articles outside their prime citation years.

\section{Methods}

\section{Classifications}

We classify publications into two categories, OA and Closed. As described above, we define OA as free to read online, either on the publisher website or in an OA repository; all articles not meeting this definition were defined as Closed. We further divide the OA literature into one of four exclusive subcategories, resulting in a five-category classification system for articles:

- Gold: Published in an open-access journal that is indexed by the DOAJ.

- Green: Toll-access on the publisher page, but there is a free copy in an OA repository.

- Hybrid: Free under an open license in a toll-access journal. 
198

199

200

201

202

203

204

205

206

207

208

209

210

211

212

213

214

215

216

217

218

219

220

221

222

223

224

225

229

230

231

232

233

- Bronze: Free to read on the publisher page, but without an clearly identifiable license.

- Closed: All other articles, including those shared only on an ASN or in Sci-Hub.

These categories are largely consistent with their use throughout the OA literature, although a few clarifications are useful. First, we (like many other OA studies) do not include ASN-hosted content as OA. Second, categories are exclusive, and publisher-hosted content takes precedence over self-archived content. This means that if an article is posted in both a Gold journal and an OA repository, we would classify it as Gold, not Green. Put another way, publisher-hosted content can "shadow" archived articles that would otherwise be Green. This definition of Green ("available in a repository but not available from the publisher") is often used in the OA literature (including by Steven Harnad, the coiner of the Green and Gold terms [Harnad et al., 2008]), but this usage is not unanimous. Some studies allow a given article to be both Gold and Green; compared to these, our classification system does undercount Green. Hybrid articles share properties with Gold articles (both are free to read and are licensed for reuse), but differ in the venue of publication (i.e., Hybrid articles are published in journals not considered open access by the DOAJ) and in that Hybrid articles are not necessarily immediately available (i.e., they may only be freely available after an embargo). We also add a novel subcategory, Bronze. Bronze shares attributes of Gold and Hybrid; like both, Bronze OA articles are publisher-hosted. Unlike Gold OA, Bronze articles are not published in journals considered open access in the DOAJ. Unlike Hybrid, Bronze articles carry no license information. Although this lack of identifiable license may not be intentional, without an identifiable license, the articles are free to read but do not allow extended reuse rights beyond reading. It is also not clear if Bronze articles are temporarily or permanently available to read for free.

Finally, we should add that, although our categories of choice reflect the OA literature, they do not necessarily reflect the more complex reality of scholarly publishing today. Organizations like SciELO and Redalyc in Latin America have been acting simultaneously as publishers and repositories and many of the articles found on their site do not fall neatly into the above categories (Packer, 2010).

\section{The oaDOI system}

We assigned the categories above by calling the oaDOI service with a DOI for each item. The oaDOI returns a link to a legally-available OA version of the article, when one is available. ${ }^{9}$ It contains records for all 88 million Crossref DOls ${ }^{10}$. The oaDOI service crawls, aggregates, normalizes, and verifies data from many sources including $\mathrm{PMC}^{11}, \mathrm{BASE}^{12}, \mathrm{DOAJ}^{13}$, and thousands of institutional repositories and publishers. The oaDOI system offers a fast, free API with no rate-limits, allowing it to support a variety of other services and tools. At the time of writing, oaDOI processes approximately 500,000 requests daily--roughly twice the daily uses of

9 https://oadoi.org/

10 DOls are short, unique identifiers for scholarly papers. Crossref is a nonprofit that helps a the DOI system, and is by far the largest supplier of academic DOls in academia.

11 https://www.ncbi.nlm.nih.gov/pmcl

12 https://www.base-search.net/about/en/

13 https://doaj.org/ 
234 Sci-Hub ${ }^{14}$ (Bohannon, 2016; Himmelstein, Romero, et al., 2017). The majority of this volume 235 comes from around 700 academic libraries, who use oaDOI to help readers find articles where 236 the library has no subscription access, addressing the discoverability problem (Chen, 2013). The 237 oaDOI service also powers the Unpaywall browser extension, which helps readers to find legal 238 OA copies of paywalled articles as they browse; Unpaywall currently has over 80,000 active 239 users. The oaDOI codebase is open source, and the service is free and open via an open API.

241 To assess the accuracy of our automated OA determination, a random subsample of 500 articles were chosen from our main "Crossref-DOI" sample, described below. We manually searched the internet for each article in our subsample to determine if the paper was freely available on the publisher's website, or on another website, such as an institutional repository, an academic social networking site, or on a personal webpage. DOls were resolved by appending the DOI to "https://doi.org/". If the full text was available through that link, articles were marked as being freely available from the publisher's site. If articles required a subscription, the title of the article was entered into Google Scholar (GS) and into Google to find alternative versions (i.e., preprints or archived copies). If the fulltext was found on any publisher page or OA repository, these were marked as being freely available from an archive. If the only available open copy was hosted on an academic social network (like Academia.edu or ResearchGate), this was noted but for the sake of the study these were not counted as any category of OA, and were instead added to the "Closed" category;

254

255

256

257

258

259

260

261

262

263

264

265

266

267

268

269

270

271

272

273

274

19
The performance of oaDOI is summarized below, compared to these manual accuracy checks. The complete dataset behind this summary is available in supplementary information. Using this data we calculated the recall and precision of the system. "Recall" asks the question, "when an article is open, how often does oaDOI correctly identify it as open?" The recall of the service is $77.0 \%$, meaning that $77 \%$ of the truly open articles are correctly identified as open by oaDOI. "Precision" asks the question, "When oaDOI says an article is open, how often is it correct?" The precision of the system is $96.6 \%$, meaning that $96.6 \%$ of the time that oaDOI reports an article is open, it really is open.

These results can be roughly compared to the recall of $86.4 \%$ and precision of $99.1 \%$ reported by Archambault et al. (2014) for their automated system. Their accuracy estimate was also calculated based on a sample of 500 data points, giving each estimate a margin of error of \pm 4.5 percentage points. The Archambault study used a narrower date window for their sample (starting in 1996, versus our Crossref-DOI sample which was not time restricted), resulting in a more homogeneous task, which may partially explain their somewhat better performance.

The oaDOI service is optimized for high precision, rather than high recall. The very high precision of oaDOI means that any estimates derived from the database can be considered a conservative estimate of the actual percentage of open access in the literature. That is, we can safely assume that when oaDOI reports a certain percentage of open access, the real percentage is at least that high-and almost certainly higher given that recall was less than perfect. Put another way, oaDOI delivers very few false positives (where it mistakenly calls an article open), but a relatively high number of false negatives (where it mistakenly calls an article

14 based on a Sci-Hub dataset released in 2016 (the most recent data available) 
275

276

277

278

279

280

281

282

283

284

285

286

287

288

289

290

291

292

293

294

295

296

297

298

299

300

301

302

303

304

305

306

307

308

closed) (Table 1). Future improvements to the system are planned that will improve recall while keeping precision high.

\section{Study samples}

Three samples of DOI-assigned scholarly resources are summarized in Table 2 and described further below.

\section{Crossref sample}

The first sample, "Crossref-DOls," is a random sample of 100,000 journal articles with Crossref DOIs, across all publication years. There are approximately 88 million Crossref DOls in total as of May 2017. In order to exclude books, datasets, and other non-article content, we sampled only items whose "type" was listed as "journal-article" in the Crossref API metadata; there are 66 million of these. To verify the accuracy of Crossref metadata, we manually checked 150 items assigned to type "journal-article," and determined that $93 \%$ were indeed journal articles; the remaining $7 \%$ were mostly journal front-matter such as tables of content or instructions to authors.

The purpose of this sample is to roughly proxy the scholarly literature as a whole. As such, it has strengths and weaknesses. One weakness is that although Crossref includes information on citation counts and discipline categorization, we found these to be quite incomplete, and therefore not useful for the present study. Another is that researchers in the scientometrics and OA fields have largely relied on other indexes, particularly Scopus and Web of Science (WoS), to represent the literature as a whole; this makes our results more difficult to compare to previous work. Finally, DOls are known to be less frequently assigned by publishers in certain disciplines (like humanities; Gorraiz 2016), in certain geographic regions (particularly the developing world), and among older articles (Boudry, 2017); consequently, these segments will be underrepresented in our sample. This said, Scopus and WoS are also known to underrepresent important segments of the literature (Mongeon \& Paul-Hus, 2016), and so this failing is not limited to Crossref. Moreover, the Crossref sample has important advantages of its own over other indexes. While no sample of the scholarly literature will be complete in every regard, the Crossref index is more expansive than other sources: in July 2017 there were 67 million journal articles indexed in Crossref compared to 30 million in Scopus ${ }^{15}$. Also, Crossref has the advantage of being entirely free and open to use, while Scopus and WoS are subscription-access databases; this allows the study data to also be free and open, promoting replication and reuse of our results in further research. However, we did turn to the subscriptionaccess WoS in order to answer questions about the discipline and citation counts of OA articles, since Crossref data is lacking in these areas.

\section{WoS sample}

310 The second sample, "WoS-DOls," is a random sample of 100,000 journal articles with DOls that

311 are indexed by Web of Science. The sample was drawn from a local version of the WoS

312 database at the Observatoire des sciences et des technologies (OST) at the Université du 
313 Québec à Montréal. Only articles that WoS defines as "citable items" are included in the sample;

314 this excludes non-peer reviewed content such as editorial material and news items. This sample

315 is restricted to articles published between 2009 and 2015, due to DOI availability constraints.

316 The sample of 100,000 articles is randomly drawn from a population of 8 million articles and

317 reviews with a DOI in WoS published between 2009 and 2015 as of May 2017.

318 Because the WoS sample is restricted to certain publication years, due to availability of DOIs in

319 the WoS database, this sample is unsuitable for estimating the proportion of the total literature

320 that is OA. However, it is more useful than the Crossref sample in some ways: the WoS sample

321 included accurate discipline information for each article (described below), and also citation

322 counts. Therefore we use the WoS sample to assess OA prevalence by discipline and also the

323 citation impact of recent OA papers. We do not encourage comparisons between the OA

324 percentages in the WoS sample and the Crossref sample, because of large differences in the

325 sampling frames.

326

327

Documents in the WoS-DOls sample were classified using the National Science Foundation

328

329

(NSF) journal classification system. This system assigns every journal exactly one "discipline" (a high-level categorization) and exactly one "specialty" (a finer-grained categorization). Because

330

331

332 this is a journal-level classification, all articles from a given journal are assigned the same discipline and specialty as the journal. A downside of this approach is that the system classifies multidisciplinary journals (e.g., Nature, PNAS, PLOS ONE) as "biomedical research," despite their publishing many articles from other fields. ${ }^{16}$ In these cases, we used a ground-up, articleby-article classification approach. Each article published in a list of multidisciplinary journals was assigned to the NSF specialty which appeared most frequently in its own reference list. In other words, papers published in multidisciplinary journals were classified at the article level (instead of at the journal level) to the subject area which they cite most frequently. ${ }^{17}$

We assess the relative impact of open and closed articles, using citations as an indicator of their scholarly impact. There are several properties of articles, however, that can confound this kind of comparison. Chief among these are the article's discipline (some fields are much more cited than others) and its age (older articles have had more time to gather citations). In order to address this, we computed a normalized expected number of citations for each article, based on its age and its NSF specialty, by comparing it to the average citations for similar articles ${ }^{18}$.

Using this approach, each article receives an average relative citation (ARC). An ARC of 1.0 indicates that a document was cited according to expectations based on documents published in the same year and NSF specialty, while an ARC above or below 1.0 indicates that the citation impact was above or below world average, respectively. Using these field-normalized citation rates, citation impact can be compared across scientific disciplines as well as across years. We can also compute mean ARCs for groups of articles, like "all open articles" or "all closed articles,"

16 These journals were identified by selecting journals with over a one thousand articles per year from those classified in the general "biomedical research" category. The full list of journals meeting these criteria were: PLOS ONE, Nature, Science, Scientific Reports, PNAS, Nature Communication, PeerJ, and Science Advances.

17 Ties between frequently cited specialties were resolved randomly; that is, if a paper cites exactly the same amount of papers from two NSF specialties, it was assigned to one of the two at random. 
350 allowing us to compare normalized impact between these two groups. Analyzing results on the 351 level of NSF disciplines, data is not shown for the Humanities $(n=1,091)$ and Arts $(n=164)$,

352 because they are underrepresented both in the Web of Science and in terms of DOI coverage.

\section{3}

354

355

356

357

358

359

360

361

362

363

364

365

366

367

368

369

370

371

372

373

374

375

376

377

378

379

380

381

382

383

384

385

386

\section{Unpaywall sample}

The third sample, "Unpaywall-DOIs," is a random sample of 100,000 articles accessed by users of the free, open-source Unpaywall browser extension, gathered over a one-week time window. We collected IP addresses and DOI requests made to the oaDOI service through the Unpaywall browser extension during the week of June 5-June 11, 2017. In that time period there were 374,703 total accesses, 213,323 unique DOls, and 42,894 unique IP addresses gathered in total, from which 100,000 unique DOls were randomly sampled.

This sample was used to assess the prevalence of OA experienced by users of the Unpaywall extension (since Unpaywall uses oaDOI data to find $\mathrm{OA}$ ). It is a convenience sample of what articles people are interested in reading, and thereby lets us roughly estimate the percent of this literature that is OA. The sample has serious limitations, however: we don't know the demographics of Unpaywall users, and we are aware of a bias towards users from the U.S. (as determined by the IP addresses). As such, we cannot accurately generalize the results by education level, discipline, or purpose in reading the scholarly literature.

\section{Results}

\section{RQ1. What percent of the literature is open access?}

\subsection{How much of the literature is OA?}

We found 27.9\% [CI95: 27.6-28.2] of all DOI-assigned journal articles are OA, using the Crossref-DOI sample. Based on this, we estimate there are 18.6 million OA articles with Crossref DOls (CI95: 18.4-18.8). This is the total population of OA articles that can be identified and accessed by oaDOI. Given our finding (described in Methods above) that the oaDOI service finds $77 \%$ of OA compared to manual searches, we can further estimate that an additional 3.5 million articles are OA but not detectable by this version of oaDOI.

People reading the literature using the Unpaywall browser extension encounter a significantly higher proportion of OA: we found that 47.0\% [CI95: 46.7-47.3] of the Unpaywall-accessed sample is open access. The main reason for this is article age: since this sample is based on the behavior of actual readers, it is disproportionately comprised of recent articles. In fact, half the accessed articles were published in the last 2 years. Recent articles are much more likely to be OA than their older counterparts (see Results section 1.3 below).

\subsection{What types of Open Access are most common?}

The proportion of OA by subtype is relatively similar across the samples, as shown in Figure 1 and Table 3. Green OA represents a relatively small percentage of OA articles in all three samples. This is partly because self-archived articles are only counted as Green where there is no publisher-hosted option available; that is, Green OA is sometimes "shadowed" by Gold, 
387 Bronze, or Hybrid articles. Bronze is the most common OA subtype in all the samples, which is 388 particularly interesting given that few studies have highlighted its role. We manually inspected a 389 small sample of Bronze articles in order to understand this subcategory more; we found that 390 while many Bronze articles were Delayed OA from toll-access publishers, nearly half were 391 hosted on journals that published $100 \%$ of content as free-to-read but were not listed on the 392 DOAJ and did not formally license content (using CC-BY or any other license). Such journals 393 might be better described as "Dark Gold" or "Hidden Gold" than Bronze. A more complete 394 examination of Bronze falls outside the scope of this study, and therefore further investigation 395 will be undertaken in future work.

396

397

398

399

400

401

402

403

404

405

406

407

408

409

410

411

412

413

414

415

416

417

418

419

420

421

422

423

424

425

426

427

\subsection{How does Open Access vary by year of publication?}

Figure 2 presents the number (left panel) and proportion (right panel) of papers by access category and publication date. Articles published in the last 20 years are increasingly OA, and this trend shows no sign of slowing. More recent articles are more likely to be OA, with the most recent year examined also containing the most OA: $44.7 \%$ of 2015 articles are OA [CI95: 43.346.2\%], including 17.6\% Bronze [CI95: 16.2-19.1], 9.4\% Hybrid [CI95: 8.0-10.9], 11.3\% Gold [CI95: 9.9-12.8], and 6.3\% Green [CI95: 4.9-7.8]. Well over one million OA papers were published in 2015. This growth trend has largely been driven by dramatic growth in Gold and Hybrid OA since the year 2000 . However, more than $20 \%$ of papers published before the digital age are also freely available. The majority of these older OA papers are Bronze, and based on their age they are probably more precisely Delayed OA, although additional investigation will be required to confirm this. Bronze OA remains remarkably constant as a proportion of the literature for all publication years examined.

The number and proportion of Green papers must be interpreted with particular caution, due to several factors. First, unlike publisher-hosted OA (Gold, Bronze, and Hybrid), the date when the Green article became open is generally different from the date the article was first published. Authors often self-archive articles years after (or before, in the case of preprints) their original publication, leading to so-called "backfilling" of Green stocks (Archambault et al., 2014). Consequently, the graph cannot show the growth of Green OA over time; this would require longitudinal analysis over several years, and so is outside the scope of this analysis. Instead it shows the number and proportion of Green OA by publication year of the article. Second, many articles cannot be legally self-archived until a certain number of months after publication; this embargoing likely influences the apparent plateau in Green shown in Figure 2. Finally, as noted earlier, many self-archived articles would otherwise be Green except for being "shadowed" by a Gold, Bronze, or Hybrid of the same article elsewhere. For more detail on the growth of shadowed Green OA, see Appendix Figure A2 and A3.

\subsection{How does Open Access vary by publisher?}

We analyzed a subset of the Crossref-DOls sample by publisher (as listed on the Crossref metadata record) to understand how the extent and types of OA are common across publishers for recent publications (between 2009 and 2015). As we can see in Figure 3 (left), the largest publishers by volume publish the most OA articles by volume, led by Elsevier. As a proportion of all articles published (Figure 3, right), however, PLOS and Hindawi distinguish themselves as 
428

429

430

431

432

433

434

435

436

437

438

439

440

441

442

443

444

445

446

447

448

449

450

451

452

453

454

455

456

457

458

459

460

461

462

463

464

465

being the only publishers in the top 20 with $100 \%$ OA. More than half of the papers published by Oxford University Press, Nature Publishing Group, IOP Publishing, and the American Physical Society (APS) are freely available online. In the case of APS this is largely driven by content available through repositories such as arXiv (for more details on repositories, see figure A1).

\subsection{How does Open Access vary across disciplines?}

We used the WoS-DOls sample to examine OA prevalence differences by discipline, because of the easy availability of discipline metadata in the WoS index. Figure 4 displays our results. More than half of the publications are freely available in biomedical research and mathematics, while in chemistry and engineering \& technology less than $20 \%$ of the papers are freely available. Figure 4 also highlights the popularity of Green OA in disciplines like physics and mathematics, where more than one fifth of papers are available only through online repositories (mainly arXiv). Hybrid articles are particularly prevalent in mathematics (9.4\%), biomedical research (8.1\%) and clinical medicine $(6.3 \%)$, while authors in biomedical research $(15.3 \%)$, health $(11.7 \%)$, mathematics $(11.2 \%)$ and clinical medicine $(10.3 \%)$ often publish in Gold journals.

Large variations can also be observed on the more detailed level of NSF specialties (Figure A5). At more than $80 \%$ of OA articles, astronomy \& astrophysics ( $87 \%)$, fertility ( $86 \%)$, tropical medicine (84\%), and embryology (83\%) were the specialties where access to literature was the most open. At the other end of the spectrum are pharmacy $(7 \%)$, inorganic \& nuclear chemistry $(7 \%)$, and chemical engineering $(9 \%)$, where publications were hidden behind a paywall for more than $90 \%$ of papers. More detail on these and other NSF specialties can be seen in Appendix Figure A5.

\section{RQ2. What is the scholarly impact of open access?}

Comparing the average relative citation impact of different access categories, the OACA is corroborated: Papers hidden behind a paywall were cited $10 \%$ below world average $(A R C=0.90)$, while those that are freely available obtain, on average, $18 \%$ more citations than what is expected $(A R C=1.18)$. However, citation impact differs between the different manners in which papers are made available for free: those that are only available as Green OA (ARC=1.33) and Hybrid OA papers $(A R C=1.31)$ are cited the most with an impact of more than $30 \%$ above expectations, those available as Bronze are cited $22 \%$ above world average, while papers published as Gold OA obtain an ARC of 0.83 . This constitutes an average relative citation impact of $17 \%$ below world average and $9 \%$ below that of articles hidden behind a paywall. Figure 5 below describes these findings.

These trends vary over time, however, as shown in Figure 6. While the ARC of closed access papers remains below world average throughout the period studied, it increased from .86 in 2009 to .93 over in 2014 and 2015. Meanwhile, when looking across all open types, the mean citation rate is consistently above the world average, fluctuating between 1.15 and 1.22. This fluctuation is guided by differences between the access types, with the impact of Hybrid OA papers increasing over the time period. While Green OA papers' mean citation rate remain 
466

467

468

469 relatively stable, the highest impact, for 2015, is obtained by Bronze and Hybrid. The only form of open for which mean impact has decreased steadily over time is Gold. The results for more recent years are only based on a short citation window, however, and results might change over the next years as citations accumulate.

\section{Discussion and Conclusion}

471 Access to scholarly literature is at the heart of current debates in the research community.

472 Research funders are increasingly mandating OA dissemination to their grantees while, at the

473 same time, the growth in toll-access subscriptions costs have prompted more and more

474 university libraries to cancel subscriptions. In this context, several tools have been developed to

475

476

477

478

479

480

481

482

483

484

485

486

487

488

489

490

491

492

493

494

495

496

497

498

499

500

501

502

503

504

505 provide access - both legally and illegally_to scholarly literature. Using data from one of these tools (oaDOI), this paper addresses two broad research questions: what percent of the literature is $O A$ and how does it vary by type of $O A$, and what is the mean scholarly impact of papers diffused through this form. Three large samples were used, to assess different aspects of OA patterns: 1) 100,000 articles that have a Crossref DOIs, which allows us to assess the relative proportion of OA across all existing literature; 2) 100,000 WoS-indexed journals articles that have a DOI, which allows us to assess the scholarly impact of OA and non OA papers; 3 ) 100,000 articles accessed by users through the Unpaywall browser extension, which lets us assess the proportion of OA papers found by users of this free tool.

We found that $28 \%$ of all journal articles are freely available online (Crossref-DOI sample). Encouragingly for proponents of OA, this proportion has been growing steadily over the last 20 years, driven particularly by growth in Gold and Hybrid. Articles from 2015, the most recent year examined, had the highest proportion OA (45\%), as well as the largest absolute number of OA articles published in a single year. This disproportionate level of OA in recent years, combined with readers' preference for more recent articles, leads to a felicitous situation for readers: the proportion of OA they experience as they browse and search is better than the overall percentage of OA across the literature as a whole. Users of the Unpaywall browser extension, which gives individual readers access to the oaDOI service, encounter OA articles nearly half $(47 \%)$ of the time. The effect almost certainly extends beyond Unpaywall users; one may assume readers in general also favor newer articles, and therefore benefit from the growth of Gold, Bronze, and Hybrid OA among recent papers, even without using Unpaywall. More studies of readership data from other sources would be useful to quantify this further.

Interestingly, we found that the majority of OA articles are Bronze-hosted on publisher websites, either without a license at all or without an open license. This is surprisingly high given that Bronze is relatively little-discussed in the OA literature, and suggests that this OA category deserves further attention from the OA community. In particular, Bronze OA may be significant in a policy context, since, unlike other publisher-hosted OA, Bronze articles do not extend any reuse rights beyond reading, making them Gratis OA. Much more research is needed into the characteristics of Bronze OA. How many Bronze articles are licensed openly, but do not make their license available? Is Bronze disproportionately non-peer-reviewed content? How much of Bronze OA is also Delayed OA? How much Bronze is Promotional, and how transient is the free- 
506 to-read status of this content? How many Bronze articles are published in "hidden gold" journals 507 that are not listed in the DOAJ? Why are these journals not defining an explicit license for their 508 content, and are there effective ways to encourage this? These and other questions are outside 509 the scope of this study but may provide fruitful insights for future OA research and policy.

510 Only about $7 \%$ of the literature overall (and $17 \%$ of the OA literature) is Green. This is may at 511 first seem disappointing, given years of advocacy focused on Green OA as well as ongoing 512 growth in the number of Green OA mandates (Björk, 2014). However, the full context of Green 513 OA provides reasons for optimism. First, many papers are archived in repositories but are not 514 counted as Green in this analysis because they are also available on the publisher site as 515 Hybrid, Gold, or Bronze versions. These "shadowed Green" copies provide a useful safety net 516 that preserves access in cases where publishers rescind it (as could potentially happen with 517 Delayed OA and other Bronze articles). Further research is needed to determine the prevalence 518 of shadowed Green OA in various disciplines. Second, the phenomenon of "backfilling" (authors 519 self-archiving content published across all years, not just the current one) means that although 520 the percentage graph of Green OA does not show the same year-over-year slope as Gold or 521 Hybrid, the line itself may be rising across all years as authors gradually self-archive papers from 522 years or even decades ago. This assumption is supported by results reported by Archambault, 523 Côté, Struck, \& Voorons (2016). Finally, the relatively low proportion of green OA encouragingly 524 leaves room for continued growth. While most journals published by major publishers (Elsevier, 525 Wiley, Springer, etc.) allow for self-archiving, research shows that only a small proportion of 526 papers from these publishers actually are self-archived in OA repositories; for example, Smith et 527 al. (in press) report using a sample of Global Health Research papers that only $39 \%$ of them 528 made use of available self-archiving rights.

529 Our results confirm the Open Access Citation Advantage found by other studies: open articles 530 receive $18 \%$ more citations than otherwise expected. While at least some of this boost is likely 531 due to the fact that more access allows more people to read and hence cite articles they 532 otherwise would not, causation is difficult to establish and there are many possible confounders. 533 Most discussed is the so-called "selection bias postulate," (Craig et al., 2007) which suggests 534 that authors choose only their most impactful work to make OA. The current study does not 535 examine the cause or directionality of correlation, but does find that it exists in a very large 536 sample that is relatively representative of the literature as a whole. Funder requirements may 537 also play a role in the observed citation advantage: high-profile funders are more likely to have 538 an OA publishing requirement; at the same time, well funded studies are independently more 539 likely to receive more citations than poorly funded studies (Berg, 2010). Interestingly, Gold 540 articles are actually cited less, likely due to an increase in the number of newer and smaller OA 541 journals. Some of these journals are from regions of the world not historically indexed by WoS, 542 are published in languages other than English, or might be considered to be less prestigious 543 because they have not had time to become established or accumulate citations (Archambault et 544 al., 2013). On the flip side, the citation disadvantage of Gold OA is likely also affected by the 545 continued growth of so-called 'mega journals' such as PLOS ONE (PLOS, n.d.). Whatever the 546 reason, the lower impact of Gold means the overall citation advantage is strongly driven by 547 Green, Hybrid, and Bronze content. In sum, while several factors can affect the observed 548 differences in citation rates, and causation remains difficult to establish, the fact remains that 549 scholars are much more likely to read and cite papers to which they have access than those that 
550 they cannot obtain. Hopefully the existence of a free, open index of OA content will help support

551 further research into the OACA question.

552 The relatively high percentage of OA found in this study, particularly among readers of the free 553 Unpaywall extension, has important potential implications for academic libraries. Increasingly, 554 these libraries are under pressure to meet growing prices of "Big Deal" subscription packages, 555 and the once-unthinkable outcome of canceling these Big Deals is becoming an increasingly 556 realistic option. In this environment, knowing that around half of the literature of interest is 557 available without any subscription may tip the scales toward cancellation for some institutions-558 particularly given that this percentage seems to be growing steadily. Indeed, the Universite de 559 Montréal's cancellation of their Taylor \& Francis subscription package (Université de Montréal, 560 2017) is particularly interesting, given that their cancellation announcement directly pointed 561 faculty to Unpaywall and other tools to help them access OA content. This may seem a radical 562 suggestion, but cancellation of subscription journals has long been part of the universal OA 563 roadmap (Anderson, 2017b). Even when the percentage of OA is not enough to support outright 564 cancellation, it may be enough to negotiate better subscription rates by supporting calculation of 565 "OA-adjusted Cost Per Access" (Antelman, 2017). However, much more study is needed to see 566 how OA availability varies across journals and Big Deal packages, along with praxis-oriented 567 work building OA analysis tools that help librarians make cancellation choices.

568 This study has several important limitations. Our dataset only includes journal articles with DOIs, 569 which means that disciplines and geographical areas which rely more heavily on conference 570 papers or articles without DOls are underrepresented. Our Crossref sample includes about $7 \%$ 571 journal "front matter" that the journal has assigned a DOI and Crossref labelled "journal article" 572 but is actually a page describing the journal Editorial Board or similar. Our Bronze OA category 573 includes articles published in OA journals which aren't indexed in DOAJ; future work must 574 identify these OA journals and classify such articles as Gold. As discussed in our definition of 575 OA, when finding open copies we ignored free-to-read articles from academic social networks 576 like ResearchGate and Academia.edu. The oaDOI system has some coverage of articles 577 published on personal web pages, but this is quite limited compared to web-scale indexes like 578 Google. The oaDOI system includes thousands of institutional and subject repositories, but there 579 are some repositories that it misses. Our accuracy checks suggest that oaDOI, and therefore 580 this study, are probably overlooking around $23 \%$ of OA otherwise discoverable using web 581 searches, meaning that estimates in reported in this paper undercount OA by approximately $58230 \%$. Finally, our approach did not detect when articles were deposited into repositories.

583 Because repositories are often backfilled with content that has been published many years ago, 584 this study does not measure any increase/decrease in prevalence of Green OA over time, but 585 only the proportion of Green OA by article publication date at the moment of data collection.

586 In addition to the empirical results obtained, this paper clearly shows the potential of the oaDOI 587 service for future research. The freely available oaDOI service provides scholars with the basis 588 for assessing and monitoring the development of access to scholarly literature on a large scale, 589 as well as the factors that affect it. For instance, our results show that the percentage of the 590 literature available as OA is growing, and that articles diffused through this form are generally 591 more cited than closed access articles. Several factors are likely to contribute to these trends; 592 however, those remain poorly understood. Combined with other datasets-such as the WoS, 593 Scopus, or Crossref-oaDOI allows one to assess at a large-scale the effects of various 
594 mandates on deposit rates, or to track the development of documents' accessibility to determine,

595 for example, when authors self-archive, or the sustainability of the promotional OA category.

596 Aggregated at the level of journals and publishing platforms, these data can also provide

597 librarians with indicators to help inform subscription cancellations and mitigate their effects. The

598 application of the oaDOI algorithm on a large scale also allows for more complete analysis of the

599 OA citation advantage across fields and time. As in Gargouri et al. (2010), confounding factors

600 could be mitigated by using article-level metadata to identify article pairs published in the same

601 journal issue, on the same topic or published by the same authors at the same time. We hope

602 that other scholars will dig deeper in those data to better understand OA dissemination and the

603 factors that drive it. This is of utmost importance for the future of scholarly communication.

604

605

606

607

608

609

610

611

612

613

\section{4}

615

616

617

618

619

620

621

622

623

624

625

626

627

628

629

630

\section{Data and code availability}

The datasets behind the analysis in this paper are openly available at

https://doi.org/10.5281/zenodo.837902 and the R statistics code can be found at https://github.com/impactstory/oadoi-paper1. The oaDOI code is open source at https://github.com/impactstory/oadoi, and information about accessing the oaDOI API and full dataset is at https://oadoi.org/api.

\section{Acknowledgements}

The authors would like to thank Dorothea Salo, Kristin Antelman, and John Sack for extensive and valuable comments on a draft of this article. The author order of JP and HP was determined by coin flip, as is their custom.

\section{References}

Anderson (2017a). When the Wolf Finally Arrives: Big Deal Cancelations in North American Libraries. Retrieved Jan 9, 2018, from https://scholarlykitchen.sspnet.org/2017/05/01/wolf-finally-arrives-big-deal-cancelationsnorth-american-libraries/

Anderson (2017b). The Forbidden Forecast: Thinking About Open Access and Library Subscriptions. Retrieved July 15, 2017, from https://scholarlykitchen.sspnet.org/2017/02/21/forbidden-forecast-thinking-open-accesslibrary-subscriptions/

Antelman, K. (2017). Leveraging the growth of open access in library collection decision making. Proceeding from ACRL 2017: At the Helm: Leading Transformation. Baltimore, MD. Retrieved from http://www.ala.org/acrl/sites/ala.org.acrl/files/content/conferences/confsandpreconfs/2017 LeveragingtheGrowthofOpenAccess.pdf

Archambault, É., Amyot, D., Deschamps, P., Nicol, A., F., Rebout, L., \& Roberge, G. (2013). Proportion of open access peer-reviewed papers at the European and world levels2004-2011. European Commission. Retrieved from http://www.science- 
632

633

634

635

636

637

638

639

640

641

642

643

644 645

646

647

648

649

650

651

652

653

654

655

656

657

658

659

660

661

662 663

664

665

666

667 668

Archambault, É., Amyot, D., Deschamps, P., Nicol, A., Provencher, F., Rebout, L., \& Roberge, G. (2014). Proportion of open access papers published in peer-reviewed journals at the European and world levels-1996-2013. European Commission. Retrieved from http://science-metrix.com/sites/default/files/science-metrix/publications/d_1.8_sm_ec_dgrtd_proportion_oa_1996-2013_v11p.pdf

Archambault, É., Côté, G., Struck, B., \& Voorons, M. (2016). Research impact of paywalled versus open access papers. Retrieved from http://www.1science.com/PDF/oaNumber_OACA_3million_paper.pdf

Berg, J. (2010, September 27). Measuring the scientific output and impact of NIGMS grants. NIGMS Feedback Loop Blog. Retrieved from https://loop.nigms.nih.gov/2010/09/measuring-the-scientific-output-and-impact-of-nigmsgrants/

Björk, B. C., Laakso M., Welling P., Paetau P. "Anatomy of green open access." Journal of the Association for Information Science and Technology 65.2 (2014): 237-250.

Björk, B. C. (2012). The hybrid model for open access publication of scholarly articles: A failed experiment? Journal of the American Society for Information Science and Technology, 63(8), 1496-1504.

Björk, B. (2016a). Hybrid open access—A longitudinal study. Journal of Informetrics 10(4), 919. https://doi.org/10.1016/j.joi.2016.08.002

Björk, B.-C. (2016b). The open access movement at a crossroad: Are the big publishers and academic social media taking over? Learned Publishing, 29(2), 131-134. https://doi.org/10.1002/leap.1021

Björk, B. C. (2017), Gold, green, and black open access. Learned Publishing, 30, 173-175. doi:10.1002/leap.1096

Björk, B. C., Welling, P., Laakso, M., Majlender, P., Hedlund, T., \& Guðnason, G. (2010, June 23). Open Access to the Scientific Journal Literature: Situation 2009. PLoS ONE, 5(6), e11273. https://doi.org/10.1371/journal.pone.0011273

Bohannon, J. (2016). Who's downloading pirated papers? Everyone. Science (New York, N.Y.), 352(6285), 508-12. Retrieved from http://www.sciencemag.org/news/2016/04/whosdownloading-pirated-papers-everyone

Boudry, C., Chartron G. (2017). Availability of digital object identifiers in publications archived by PubMed. Scientometrics March 110(3): 1453-1469. doi:10.1007/s11192-016-2225-6

Chan, L., Cuplinskas, D., Eisen, M., Friend, F., Genova, Y., Guédon, J., . . Velterop, J. (2002, February 14). Budapest open access initiative. Retrieved from http://www.budapestopenaccessinitiative.org/read

Chawla D. Publishers take ResearchGate to court, alleging massive copyright infringement. Science (80- ). 2017 Oct 6; 
669 Chen, X. (2013). Journal article retrieval in an age of Open Access: How journal indexes indicate

$670 \quad$ Open Access articles. Journal of Web Librarianship, 7(3), 243-254.

$671 \quad$ http://dx.doi.org/10.1080/19322909.2013.795426

672 Chen, X., \& Olijhoek, T. (2016). Measuring the Degrees of Openness of Scholarly Journals with

673 the Open Access Spectrum (OAS) Evaluation Tool. Serials Review, 42(2), 108-115.

674 https://doi.org/10.1080/00987913.2016.1182672

675 Craig, I. D., Plume, A. M., McVeigh, M. E., Pringle, J., \& Amin, M. (2007). Do open access

676 articles have greater citation impact? Journal of Informetrics, 1(3), 239-248.

$677 \quad$ https://doi.org/10.1016/j.joi.2007.04.001

678 Creative Commons. (n.d.) Attribution 4.0 International (CC BY 4.0). Retrieved from

679 https://creativecommons.org/licenses/by/4.0/

680

Davis, P. M., and Walters, W. H. (2011). The Impact of Free Access to the Scientific Literature: A

681 Review of Recent Research. Journal of the Medical Library Association 99.

682 https://doi.org/10.3163/1536-5050.99.3.008

683

Davis, P. M. (2011). Open access, readership, citations: a randomized controlled trial of scientific 684 journal publishing. FASEB Journal 25:2129-2134. https://doi.org/10.1096/fj.11-183988

685

Fortney, K. \& Gonder, J. (2015, December 1). A social networking site is not an open access

686

687

688 repository. Office of Scholarly Communication: University of California. Retrieved from http://osc.universityofcalifornia.edu/2015/12/a-social-networking-site-is-not-an-open-

689

690

Gargouri Y, Hajjem C, Larivière V, Gingras Y, Carr L, Brody T, Harnad, S. (2010) Self-Selected or

691 Mandated, Open Access Increases Citation Impact for Higher Quality Research. PLoS ONE 5(10), e13636. https://doi.org/10.1371/journal.pone.0013636

692

693

Gargouri, Y., Larivière, V., Gingras, Y., Carr, L., \& Harnad, S. (2012). Green and gold open access percentages and growth, by discipline. arXiv preprint. arXiv:1206.3664.

694

Gorraiz, J., Melero-Fuentes D., Gumpenbergera C., Valderrama-Zuriánc J-C. (2016) Availability

695 of digital object identifiers (DOIs) in Web of Science and Scopus. Journal of Informetrics

696 10(1): 98-109. doi: 10.1016/j.joi.2015.11.008

697

Greshake B. (2017). Looking into Pandora's Box: The Content of Sci-Hub and its Usage [version 1; referees: 2 approved, 2 approved with reservations]. F1000Research, 6(541)

699 http://dx.doi.org/10.12688/f1000research.11366.1

700

Hajjem, C., Harnad, S., \& Gingras, Y. (2006). Ten-Year Cross-Disciplinary Comparison of the

701 Growth of Open Access and How it Increases Research Citation Impact.

702 arXiv:cs/0606079. Retrieved from http://arxiv.org/abs/cs/0606079 
703

704

705

706

707

708

709

710

711

712

713

714

715

716

717

718

719

720

721

722

723

724

725

726

727

728

729

730

731

732

733

734

735

736

737

738

Hanlon, A., \& Ramirez, M. (2011). Asking for permission: A survey of copyright workflows for institutional repositories. portal: Libraries and the Academy 11(2), 683-702. The Johns Hopkins University Press. https://doi.org/10.1353/pla.2011.0015

Harnad, S., Brody, T., Vallières, F., Carr, L., Hitchcock, S., Gingras, Y., . . Hilf, E. R. (2004). The access/impact problem and the green and gold roads to open access. Serials Review, 30(4), 310-314. doi:10.1016/j.serrev.2004.09.013

Harnad, S., Brody, T., Vallières, F., Carr, L., Hitchcock, S., Gingras, Y., ... Hilf, E. R. (2008). The Access/Impact Problem and the Green and Gold Roads to Open Access: An Update. Serials Review, 34(1), 36-40. https://doi.org/10.1080/00987913.2008.10765150

Himmelstein, D. S., Romero, A. R., McLaughlin, S. R., Tzovaras, B. G., \& Greene, C. S. (2017). Sci-Hub provides access to nearly all scholarly literature (No. e3100v1). PeerJ Preprints. https://doi.org/10.7287/peerj.preprints.3100v1

Jamali, H. R. (2017). Copyright compliance and infringement in ResearchGate full-text journal articles. Scientometrics, 112(1), 241-254. https://doi.org/10.1007/s11192-017-2291-4

Laakso, M., \& Björk B. C. (2012) Anatomy of open access publishing: a study of longitudinal development and internal structure. BMC Medicine, 10(124). doi:10.1186/1741-7015-10124

Laakso, M., \& Björk, B. (2013). Delayed open access: An overlooked high $\square$ impact category of openly available scientific literature. Journal of the American Society for Information Science and Technology, 64(7), 1323-1329. doi:10.1002/asi.22856

Laakso, M., Welling, P., Bukvova, H., Nyman, L., Björk, B. C., \& Hedlund, T. (2011). The Development of Open Access Journal Publishing from 1993 to 2009. PLoS ONE. 6(6), e20961. https://doi.org/10.1371/journal.pone.0020961

McCabe, M., \& Snyder, C. (2014). Identifying the effect of open access on citations using a panel of science journals. Economic Inquiry, 52(4), 1284-1300. doi:10.1111/ecin.12064

McKiernan, E., Bourne, P., Brown, C., Buck, S., Kenall, A., Lin, J., . . Rodgers, P. (2016). How open science helps researchers succeed. ELife, 5. https://doi.org/10.7554/eLife.16800.

Moed, H. F. (2007), The effect of "open access" on citation impact: An analysis of ArXiv's condensed matter section. Journal of the American Society for Information Science and Technology, 58(13): 2047-2054. doi: 10.1002/asi.20663

Mongeon P. \& Paul-Hus A. (2016). The journal coverage of Web of Science and Scopus: a comparative analysis. Scientometrics. 106(1):213-28.

Morrison, H., Salhab, J., Calvé-Genest, A., \& Horava, T. (2015). Open Access Article Processing Charges: DOAJ Survey May 2014. Publications, 3(1), 1-16. https://doi.org/10.3390/publications3010001 
739

740

741

742

743

744

745

746

747

748

749

750

751

752

753

754

755

756

757

758

759

760

761

762

763

764

765

766

767

768

769

770

771

772

773

774

775

776

Ottaviani, J. (2016) The post-embargo open access citation advantage: It exists (probably), it's modest (usually), and the rich get richer (of course). PLOS ONE, 11(8), e0159614. http://doi.org/10.1371/journal.pone.0159614

Packer, A. L. (2010). The SciELO Open Access: A Gold Way from the South. Canadian Journal of Higher Education, 39(3), 111.

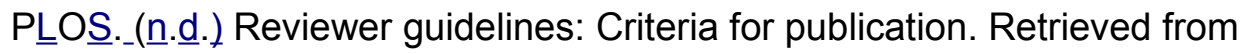
http://journals.plos.org/plosone/s/reviewer-guidelines\#loc-criteria-for-publication

Schiermeier, Q., \& Mega, E. R. (2017). Scientists in Germany, Peru and Taiwan to lose access to Elsevier journals. Nature News, 541(7635), 13. https://doi.org/10.1038/nature.2016.21223

Smith, E., Haustein, S., Mongeon, P., Fei, S., Ridde, V., \& Larivière, V. (in press). Knowledge sharing in global health research; the impact, uptake and cost of open access to scholarly literature. BMC Health Research Policy and System.

SPARC Europe. (2015). The open access citation advantage: List of studies until 2015. Retrieved from: http://sparceurope.org/what-we-do/open-access/sparc-europe-openaccess-resources/open-access-citation-advantage-service-oaca/oaca-list/

Suber, P. (2008, August 2). Gratis and libre open access. SPARC Open Access Newsletter, 124. Retrieved from https://dash.harvard.edu/handle/1/4322580

Suber, P. (2012a). Open Access. MIT Press. http://bit.ly/oa-book

Suber, P. (2012b, June 2). The rise of libre open access. SPARC Open Access

Newsletter, 164. Retrieved from http://legacy.earlham.edu/ peters/fos/newsletter/06-0212.htm

Tennant, J. P., Waldner, F., Jacques, D.C., Masuzzo, P., Collister, L. B, \& Hartgerink, C. H. (2016). The academic, economic and societal impacts of Open Access: An evidencebased review [version 3; referees: 3 approved, 2 approved with reservations]. F1000Research, 5(632), doi:10.12688/f1000research.8460.3

Tennant, J. (n.d.). The Open Access Citation Advantage. Retrieved August 2, 2017, from https://www.scienceopen.com/search\#\%7B\%22order\%22\%3A0\%2C\%22context \%22\%3A\%7B\%22collection\%22\%3A\%7B\%22id\%22\%3A\%22996823e0-8104-4490b26a-f2f733f810fb \%22\%2C\%22kind \%22\%3A0\%7D\%2C\%22kind\%22\%3A11\%7D\%2C \%22kind\%22\%3A77\%7D, doi: 10.14293/S2199-1006.1.SOR-EDU.CLPDPZB.v1

Universitât_Konstanz.._(2014, March 26 6 )._Teurer als die Wissenschaft erlaubt. Retrieved from https://www.uni-konstanz.de/universitaet/aktuelles-und-medien/aktuellemeldungen/aktuelles/aktuelles/teurer-als-die-wissenschaft-erlaubt/

Université de Montréal. (2017, May 4). UdeM Libraries cancel Big Deal subscription to 2,231 periodical titles published by Taylor \& Francis Group. Retrieved from http://www.bib.umontreal.ca/communiques/20170504-DC-annulation-taylor-francisva.htm 
777 Wagner, A.B. (2010). Open access citation advantage: An annotated bibliography. Issues in

778 Science and Technology Librarianship, 60(2). http://doi.org/10.5062/F4Q81B0W

779 Walker, T. J., \& Soichi, transl. T. (1998). Free Internet Access to Traditional Journals. Journal of 780 Information Processing and Management, 41(9), 678-694.

$781 \quad$ https://doi.org/10.1241/johokanri.41.678

782 Willinsky J. (2003). The nine flavours of open access scholarly publishing. J Postgrad Med

783 49:263-7. Retrieved from: http://www.jpgmonline.com/text.asp?2003/49/3/263/1146

784 Willinsky, J. (2009). The Access Principle: The Case for Open Access to Research and 785 Scholarship (1 edition). Cambridge, Mass.; London: The MIT Press. 


\section{Table 1 (on next page)}

Accuracy of the prototype version of the oaDOI service used in this study 
1

\begin{tabular}{|c|c|c|c|}
\hline & $\begin{array}{l}\text { oaDOI reports } \\
\text { Open }\end{array}$ & $\begin{array}{l}\text { oaDOI reports } \\
\text { Closed }\end{array}$ & $\begin{array}{l}\text { Manual count Total } \\
\text { (ground truth) }\end{array}$ \\
\hline Open & 144 & 43 & 187 \\
\hline Closed & 5 & 308 & 313 \\
\hline Total & 149 & 351 & 500 \\
\hline
\end{tabular}

2

3 


\section{Table 2 (on next page)}

Summary of samples used in this study 
1

\begin{tabular}{|l|l|l|l|c|}
\hline Sample name & $\begin{array}{l}\text { Sample } \\
\text { size }\end{array}$ & $\begin{array}{l}\text { Population } \\
\text { sampled }\end{array}$ & Purpose & $\begin{array}{l}\text { Population } \\
\text { size }\end{array}$ \\
\hline Crossref-DOls & 100,000 & $\begin{array}{l}\text { All journal articles } \\
\text { with Crossref DOls, } \\
\text { all years. }\end{array}$ & $\begin{array}{l}\text { Estimate percentage of } \\
\text { the literature that is OA. }\end{array}$ & $66,560,153$ \\
\hline WoS-DOIs & 100,000 & $\begin{array}{l}\text { All citable WoS } \\
\text { articles with DOls, } \\
2009-2015 .\end{array}$ & $\begin{array}{l}\text { Estimate citation impact of } \\
\text { recent OA papers, and } \\
\text { also OA prevalence by } \\
\text { discipline. }\end{array}$ & $8,083,613$ \\
\hline Unpaywall-DOls & 100,000 & $\begin{array}{l}\text { All articles accessed } \\
\text { by Unpaywall users } \\
\text { over a 1-week } \\
\text { period in 2017. }\end{array}$ & $\begin{array}{l}\text { Estimate percentage of } \\
\text { OA experienced by users } \\
\text { of the Unpaywall } \\
\text { extension. }\end{array}$ & 213,323 \\
\hline
\end{tabular}

2

3 


\section{Table 3(on next page)}

Percent of the literature that is $\mathrm{OA}$, by type, in three samples of 100,000 journal articles, with $95 \%$ confidence intervals. 
1

\begin{tabular}{|c|r|r|r|r|r|r|}
\hline $\begin{array}{l}\text { Access } \\
\text { Type }\end{array}$ & \multicolumn{2}{|c|}{\begin{tabular}{c}
\multicolumn{1}{c|}{ Crossref-DOI } \\
All journal articles with \\
Crossref DOls, all years. \\
("Articles with DOls" in Fig.1)
\end{tabular}} & $\begin{array}{l}\text { Wll citable WoS } \\
\text { articles with DOls, } \\
2009-2015 .\end{array}$ & $\begin{array}{l}\text { Unpaywall-DOls } \\
\text { All articles accessed by } \\
\text { Unpaywall users over a } \\
1-\text { week period in 2017 }\end{array}$ \\
\hline $\begin{array}{l}\text { OA (all } \\
\text { types) }\end{array}$ & $27.9 \%$ & $27.6-28.2$ & $36.1 \%$ & $36.0-36.2$ & $47.0 \%$ & $46.7-47.3$ \\
\hline Bronze OA & $16.2 \%$ & $16.0-16.5$ & $12.9 \%$ & $12.6-13.2$ & $15.3 \%$ & $15.0-15.6$ \\
\hline Hybrid OA & $3.6 \%$ & $3.3-3.9$ & $4.3 \%$ & $4.0-4.6$ & $8.3 \%$ & $8.0-8.6$ \\
\hline Gold OA & $3.2 \%$ & $2.9-3.5$ & $7.4 \%$ & $7.1-7.7$ & $14.3 \%$ & $14.0-14.6$ \\
\hline Green OA & $4.8 \%$ & $4.5-5.1$ & $11.5 \%$ & $11.2-11.8$ & $9.1 \%$ & $8.8-9.4$ \\
\hline Closed & $72.0 \%$ & $71.8-72.4$ & $63.9 \%$ & $63.8-64.0$ & $53.0 \%$ & $52.7-53.3$ \\
\hline
\end{tabular}

2 
Figure 1

Percent of articles by OA status, Crossref-DOIs sample vs Unpaywall-DOIs sample.

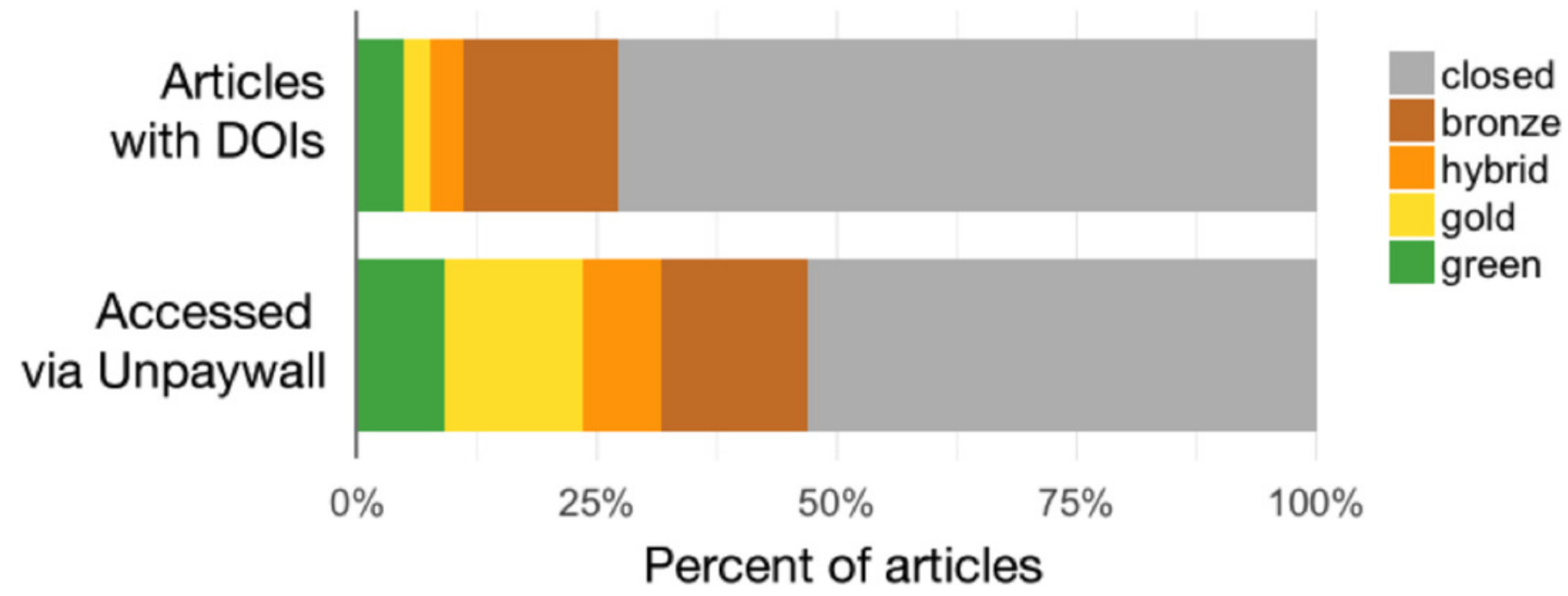




\section{Figure 2}

Number of articles (A) and proportion of articles (B) with OA copies, estimated based on a random sample of 100,000 articles with Crossref DOls

A.

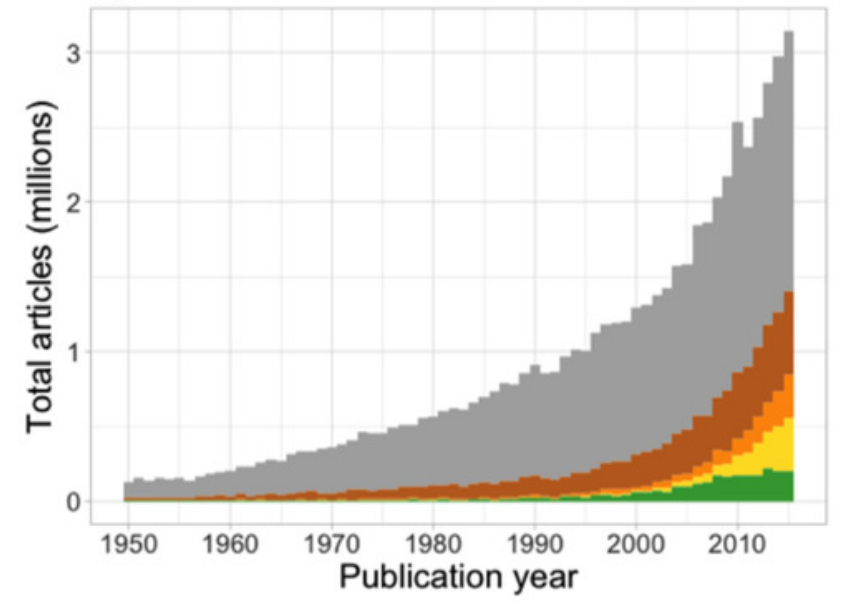

B.

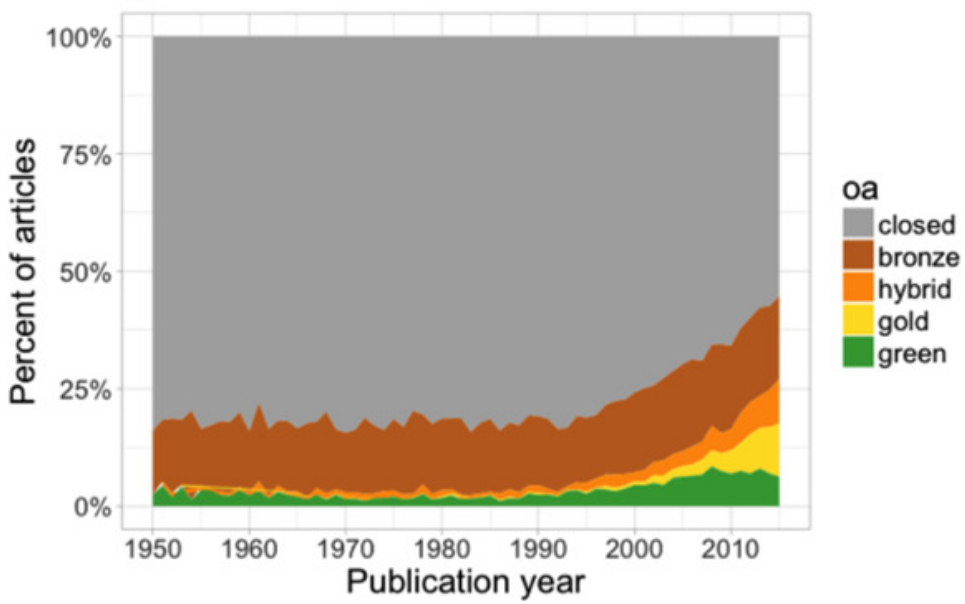


Figure 3

Number (A) and proportion (B) of articles with OA copies, by publisher, for the 20 most prolific publishers.Based on sample of 27,894 Crossref DOI-assigned articles published between 2009-2015.

A.

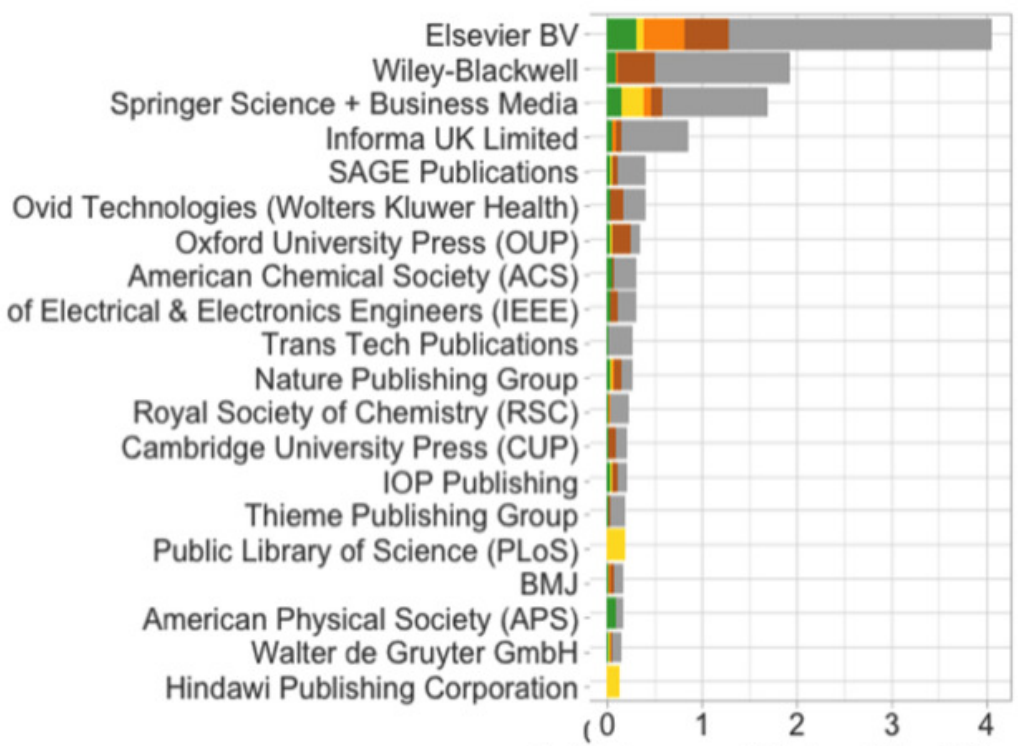

B.

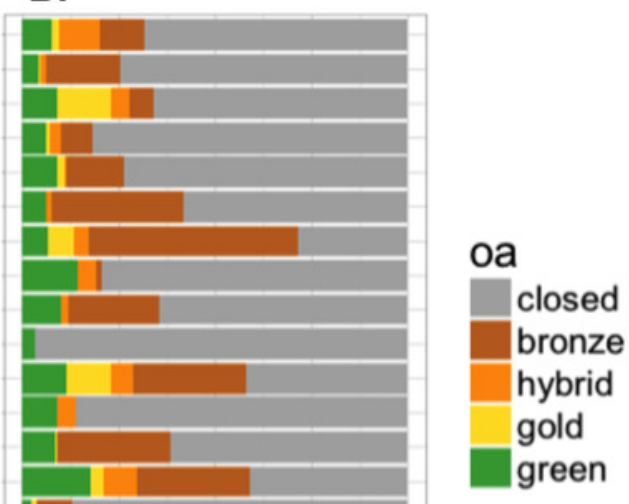

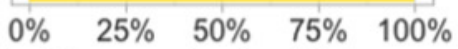




\section{Figure 4}

Percentage of different access types of a random sample of WoS articles and reviews with a DOI published between 2009 and 2015 per NSF discipline (excluding Arts and Humanities).

Biomedical Research $(n=11,360)$

Mathematics $(n=3,303)$

Clinical Medicine $(n=27,066)$

Health $(n=2,121)$

Earth and Space $(n=5,975)$

Biology $(n=6,327)$

Physics $(n=9,547)$

Psychology $(n=2,257)$

Social Sciences $(n=3,143)$

Professional Fields $(n=2,418)$

Engineering and Technology $(n=14,831)$

Chemistry $(n=10,397)$

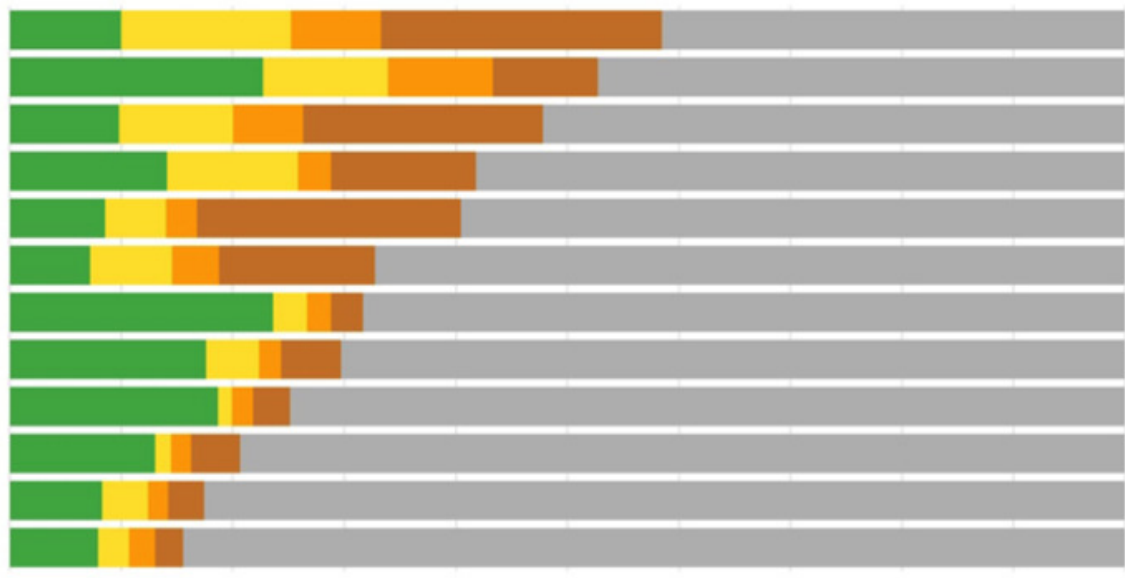

$\begin{array}{lllllllllll}0 \% & 10 \% & 20 \% & 30 \% & 40 \% & 50 \% & 60 \% & 70 \% & 80 \% & 90 \% & 100 \%\end{array}$

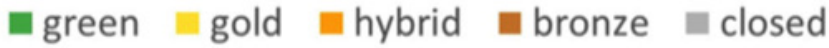


Figure 5

Average relative citations of different access types of a random sample of WoS articles and reviews with a DOI published between 2009 and 2015.

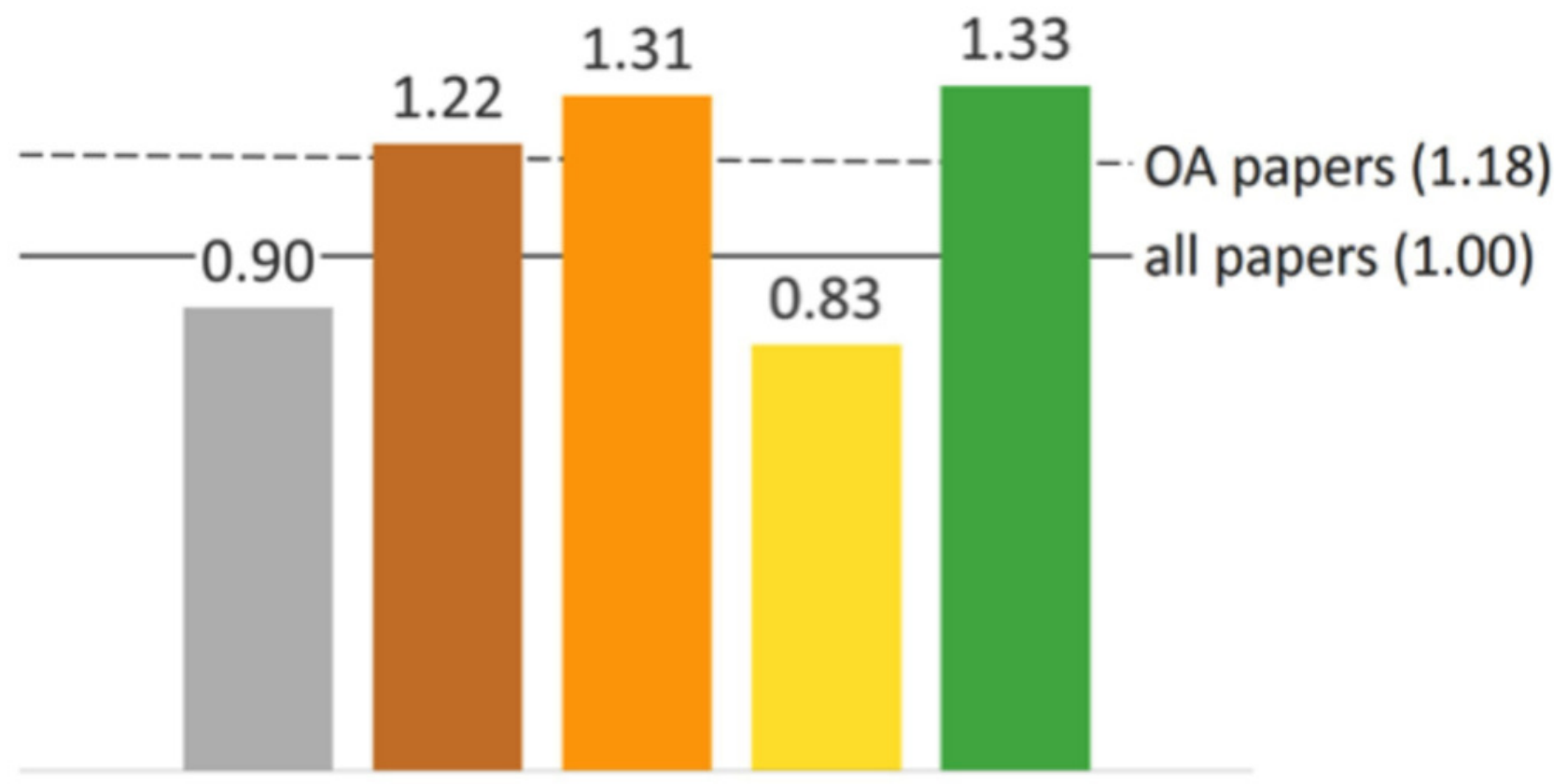

\section{Average relative citations}

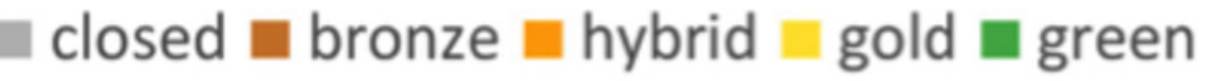


Figure 6

Percentage and impact of different access types of a random sample of WoS articles and reviews with a DOI, by year of publication.

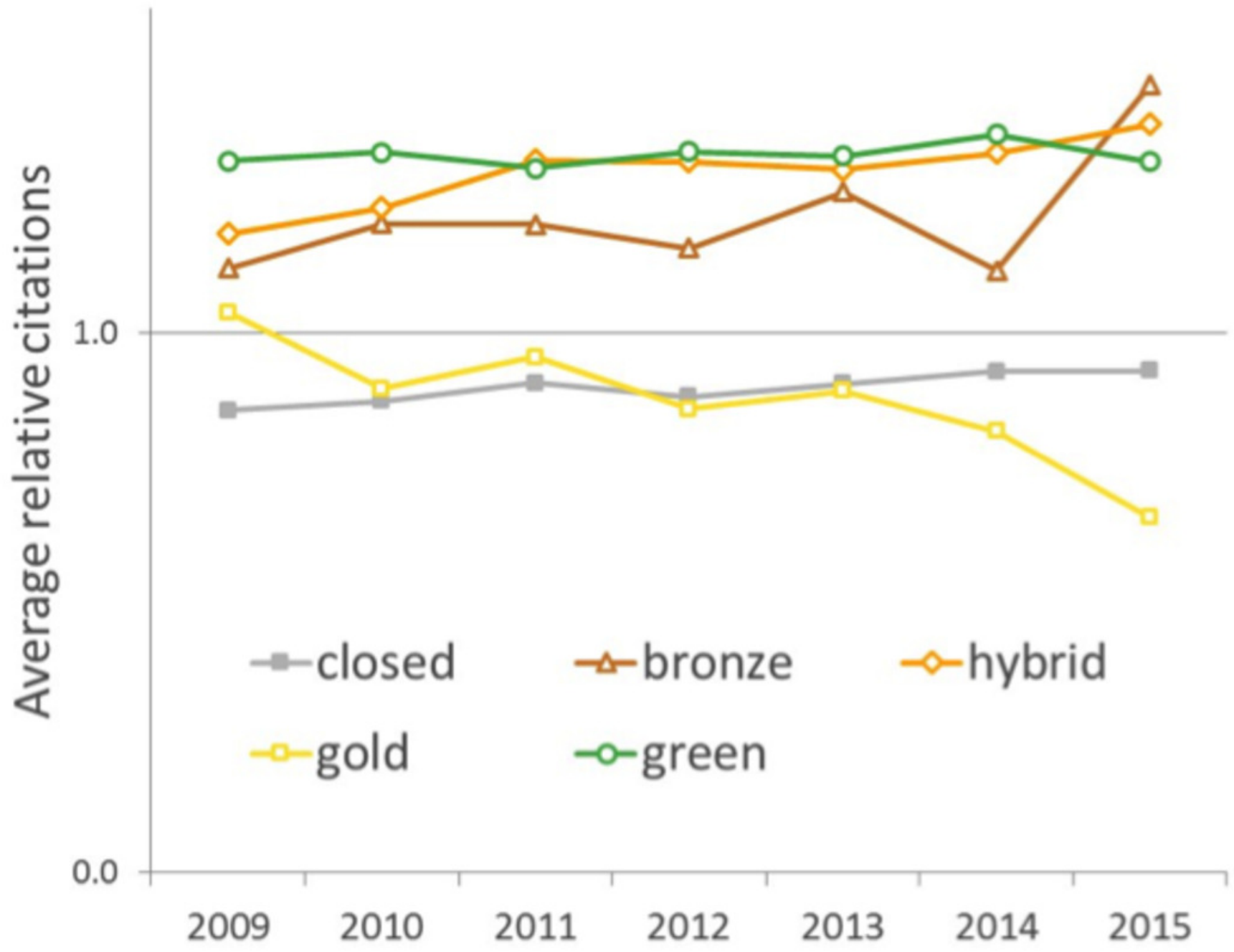

